\title{
Article
}

\section{Oral Administration of Water Extract from Euglena gracilis Alters the Intestinal Microbiota and Prevents Lung Carcinoma Growth in Mice}

\author{
Deepa Upreti ${ }^{1,+}$, Susumu Ishiguro ${ }^{1,+} \mathbb{D}$, Nicole Robben ${ }^{1}$, Ayaka Nakashima ${ }^{2}$, Kengo Suzuki ${ }^{2}$, Jeffrey Comer ${ }^{1 \mathbb{D}}$ \\ and Masaaki Tamura ${ }^{1, *}$
}

check for

updates

Citation: Upreti, D.; Ishiguro, S.;

Robben, N.; Nakashima, A.; Suzuki,

K.; Comer, J.; Tamura, M. Oral

Administration of Water Extract from

Euglena gracilis Alters the Intestinal

Microbiota and Prevents Lung

Carcinoma Growth in Mice. Nutrients

2022, 14, 678. https://doi.org/

$10.3390 /$ nu14030678

Academic Editor: Julia Schipke

Received: 26 December 2021

Accepted: 4 February 2022

Published: 5 February 2022

Publisher's Note: MDPI stays neutral with regard to jurisdictional claims in published maps and institutional affiliations.

Copyright: (C) 2022 by the authors. Licensee MDPI, Basel, Switzerland. This article is an open access article distributed under the terms and conditions of the Creative Commons Attribution (CC BY) license (https:// creativecommons.org/licenses/by/ $4.0 /)$.
1 Department of Anatomy \& Physiology, Kansas State University College of Veterinary Medicine, Manhattan, KS 66506, USA; deepa07@vet.k-state.edu (D.U.); isusumu@vet.k-state.edu (S.I.); ndrobben@vet.k-state.edu (N.R.); jeffcomer@ksu.edu (J.C.)

2 Euglena Co., Ltd., Minato-ku, Tokyo 108-0014, Japan; nakashima@euglena.jp (A.N.); suzuki@euglena.jp (K.S.)

* Correspondence: mtamura@vet.k-state.edu; Tel.: +1-(785)-532-4825; Fax: +1-(785)-532-4557

+ These authors contributed equally to this paper.

\begin{abstract}
The antitumor effects of a partially purified water extract from Euglena gracilis (EWE) and EWE treated by boiling (bEWE) were evaluated using orthotopic lung cancer syngeneic mouse models with Lewis lung carcinoma (LLC) cells. Daily oral administration of either EWE or bEWE started three weeks prior to the inoculation of LLC cells significantly attenuated tumor growth as compared to the phosphate buffered saline (PBS) control, and the attenuation was further enhanced by bEWE. The intestinal microbiota compositions in both extract-treated groups were more diverse than that in the PBS group. Particularly, a decrease in the ratio of Firmicutes to Bacteroidetes and significant increases in Akkermansia and Muribaculum were observed in two types of EWE-treated groups. Fecal microbiota transplantation (FMT) using bEWE-treated mouse feces attenuated tumor growth to an extent equivalent to bEWE treatment, while tumor growth attenuation by bEWE was abolished by treatment with an antibiotic cocktail. These studies strongly suggest that daily oral administration of partially purified water extracts from Euglena gracilis attenuates lung carcinoma growth via the alteration of the intestinal microbiota.
\end{abstract}

Keywords: natural product; water extract from Euglena gracilis; lung cancer prevention; gut microbiota alteration; fecal microbiota transplantation

\section{Introduction}

Lung cancer is a commonly diagnosed cancer type in both sexes and the leading cause of cancer-related deaths, comprising one in five cancer-related deaths worldwide [1]. Furthermore, lung cancer remains a leading cause of cancer-related death even among individuals who have never smoked [2,3]. Although many new strategies for cancer prevention and treatment are being implemented, lung cancer is predicted to cause the greatest number of deaths in 2021, in comparison to other cancer types [4]. In addition, many cancers tend to metastasize in the lungs [5], resulting in life-threatening conditions. Therefore, novel prevention and therapeutic strategies for lung cancer are urgently needed.

Euglena gracilis (E. gracilis) is a single-celled, flagellated, fast-proliferating microalga that is rich in nutrients [6]. This alga can be found in both fresh and saltwater and possesses characteristics of both plants and animals [7]. E. gracilis is used as a nutritional dietary supplement because it is rich in carbohydrates, amino acids, minerals, chlorophyll, and vitamins [8]. E. gracilis also serves as an excellent source of valuable substances such as paramylon (a water-insoluble granule or linear form of $\beta-1,3$-glucan), tocopherol, and carotenoids with pharmaceutical potentials [9]. E. gracilis with paramylon is known to have an immunomodulatory effect in both humans and animals, playing a role as an 
immunostimulant or an immunopotentiator [10,11]. Studies have revealed that E. gracilis has potential as a therapeutic due to its antimicrobial [12], antioxidant [13], anticancer [14], antiviral [15-17], and antihyperglycemic properties [18]. Furthermore, our previous study showed that the partially purified extract derived from E. gracilis prevents lung carcinoma growth in mice by stimulating host antitumor immunity through the attenuation of myeloidderived suppressor cell (MDSC) populations [19].

The gut is colonized by complex bacterial populations, many of which are beneficial for the health to the host, including protection against pathogenic microbiota, production of vitamins, absorption of ions, etc. [20]. More importantly, the gut microbiota is essential for the development, training, and function of the immune system both locally and at distant sites [21,22]. Moreover, gut microbiota-derived metabolites, such as short-chain fatty acids (SCFA), can influence host immune responses [23]. Emerging evidence demonstrates vital crosstalk between the gut microbiota and the lung through a shared mucosal immune system termed the "gut-lung axis" [21], highlighting the impact of gut microbes in lung diseases such as asthma, chronic obstructive pulmonary disease, and lung cancer [24]. In fact, there is a significant difference in the gut bacterial composition of lung cancer patients in comparison to healthy controls [25]. These studies highlight the potential of manipulating the gut microbiota and/or metabolites as a therapeutic approach for lung diseases.

Our previous finding that the oral administration of partially purified extracts of E. gracilis prevents the growth of lung cancer in mice indicates a possible interaction along the gut-lung axis [19]. Another study reported that the components of E. gracilis stimulate the growth of gut microbes such as Faecalibacterium to improve digestive health by producing butyrate, an SCFA [26]. Here we examine the mechanism behind the prevention of lung carcinoma in mice upon daily administration of E. gracilis water extract. We find that the oral administration of Euglena water extract alters the gut microbiota and plays a role in the attenuation of lung carcinoma growth. Our findings indicate that water extract from E. gracilis could be used as a prebiotic for the prevention of lung cancer.

\section{Materials and Methods}

\subsection{Animals}

Wild-type female C57BL/6 mice were purchased from Charles River Laboratories International, Inc. and were housed in a clean facility under controlled conditions of temperature $\left(20-26^{\circ} \mathrm{C}\right)$, with $30-70 \%$ relative humidity and light (12:12 h light-dark cycles). All mice were held for a week to acclimatize before the treatment. All mice were housed humanely according to university, state, and federal guidelines (AAALAC) in the AAALACaccredited animal resource facilities of the Kansas State University College of Veterinary Medicine. The condition of the mice was observed daily, and body weights were measured every three days. All experiments were carried out under approvals of the Kansas State University Institutional Animal Care and Use Committee (Protocol \# 4346) and Institutional Biosafety Committee (Registration \# 1317).

\subsection{Preparation of Extracts from E. gracilis}

Dried powder of E. gracilis was obtained from Euglena Co., Ltd. (Tokyo, Japan). Water extracts of E. gracilis were prepared by suspending this dried powder in sterile phosphate buffered saline (PBS) and incubating it at $37^{\circ} \mathrm{C}$ for $30 \mathrm{~min}$ with periodic sonication for $30 \mathrm{~s}$. The whole suspension was centrifuged at a low-speed of $34 \times g$ for $1 \mathrm{~min}$. While the precipitate was separated and used to prepare paramylon water extract (PWE), the supernatant was used to prepare the Euglena water extract (EWE) and boiled Euglena water extract (bEWE). The precipitate was suspended in PBS, followed by centrifugation at $1240 \times g$ for $10 \mathrm{~min}$. To obtain the substance denoted PWE, the precipitate derived from the first low speed centrifugation was boiled for $10 \mathrm{~min}$, followed by a high-speed centrifugation at $11,405 \times g$ for $10 \mathrm{~min}$. The resulting supernatant was used as the PWE. To obtain EWE and bEWE, the supernatant was centrifuged at $11,405 \times g$ for $10 \mathrm{~min}$ and then filtered by a $0.22 \mu \mathrm{m}$ sterile disk filter (Midwest Scientific, Valley Park, MO, USA). 
The difference between EWE and bEWE is that a boiling process was carried out before the filtration step during the preparation of bEWE. The dry weight was calculated after drying a $300 \mu \mathrm{L}$ aliquot at $55^{\circ} \mathrm{C}$ for $48 \mathrm{~h}$ from which the dry weight of PBS was subtracted $(0.125 \mathrm{mg}$ dry powder $/ \mathrm{mL})$. All the partially purified water extracts from E. gracilis were stored at $-20{ }^{\circ} \mathrm{C}$ until further use.

\subsection{Cell Culture}

The mouse Lewis lung carcinoma (LLC) cell line (CRL-1642) was purchased from American Type Culture Collection (ATCC, Manassas, VA, USA). Dulbecco's Modified Eagle's Medium (DMEM) was obtained from Mediatech, Inc. (Manassas, VA, USA). Fetal bovine serum (FBS) was obtained from Biowest (Riverside, MO, USA). The penicillinstreptomycin stock was obtained from Lonza Rockland, Inc. (Allendale, NJ, USA). The cells were cultured at $37^{\circ} \mathrm{C}$ in a humidified air atmosphere containing $5 \% \mathrm{CO}_{2}$. The cell line was authenticated by short tandem repeat (STR) DNA profiling. Both the cells were maintained in low passages $(<15)$ for this study.

\subsection{Treatment of Mice with Euglena Extracts via Drinking Water}

The effect of oral administration of EWE and bEWE on lung cancer growth was evaluated using an orthotopic LLC cell syngeneic model in C57BL/ 6 mice $(n=4-5)$. Four treatment regimens were used: (1) PBS control, (2) 150-250 mg/kg/mouse/day EWE, (3) $150-250 \mathrm{mg} / \mathrm{kg} / \mathrm{mouse} /$ day bEWE, (4) $15-25 \mathrm{mg} / \mathrm{kg} / \mathrm{mouse} /$ day PWE (Figure 1A). The extracts were orally administered daily by drinking water three weeks before LLC cell inoculation via tail vein injection $\left(2 \times 10^{6}\right.$ cells $/$ mouse). Consumption of drinking water containing extracts was monitored by measuring the weight of the water pouches every three days. Based on the average water consumption (Figure S2), doses of each extract were adjusted to maintain the desired dosage. Each mouse was monitored daily for signs of illness. Three weeks after LLC cell injection, all mice were sacrificed by cervical dislocation following exposure to saturated $\mathrm{CO}_{2}$. The lung, spleen, both of the kidneys, liver, and intestine were collected to examine their weights and fixed in $10 \%$ formalin for histological analysis. The tumor weights in the lungs were calculated by subtracting the average weight of an age-matched normal mouse lung $(142.3 \pm 11.9, n=5)$.

A

EWE, bEWE (150-250 mg/kg) PWE (15-25mg/kg)

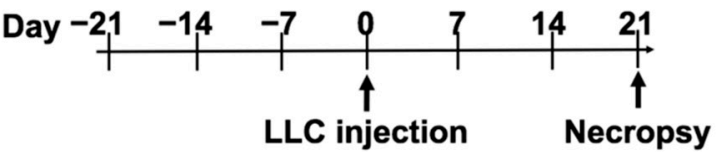

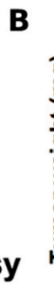

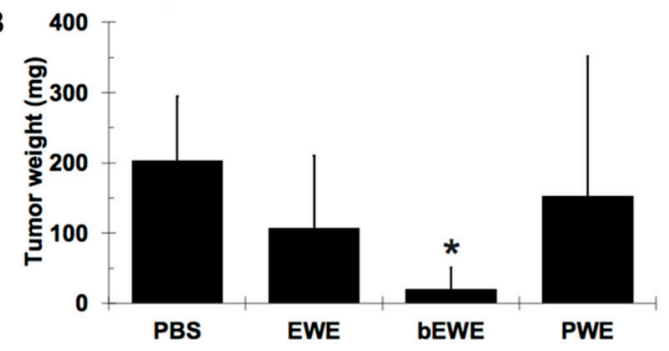

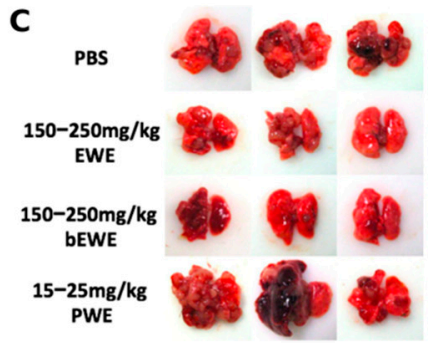

Figure 1. Pre-treatment with Euglena water extract (EWE) and boiled Euglena water extract (bEWE) attenuated growth of Lewis lung carcinoma (LLC) tumors in murine lungs. (A) Schematic illustration of the study design. (B) Average tumor weight in each treatment group; phosphate buffered saline (PBS) served as a control. (C) Macroscopic views of the lungs from LLC tumor-bearing mice in PBS or extract pretreatment groups. The scale bar in each picture represents $5 \mathrm{~mm}$. Results are presented as mean $\pm \mathrm{SD}(n=5)$. $^{*}: p<0.05$ as compared to the PBS group. PWE: paramylon water extract.

\subsection{Fecal Sample Collection and $16 S$ rRNA Amplicon Sequencing}

Feces were collected from mice receiving each of the Euglena water extracts (EWE, bEWE, or PBS), at three time-points; Day 0 (before the treatment), Day 21 (before LLC cell injection), and Day 42 (before necropsy) and kept at $-80^{\circ} \mathrm{C}$ for later use. DNA was isolated from fecal pellets $(200 \mathrm{mg}$ ) from the treated groups using the DNeasy PowerSoil Kit (Qiagen, Germantown, MD, USA). Genomic DNA samples were quantified using the Nanodrop system (Thermo Scientific, Waltham, MA, USA) and kept at $-80{ }^{\circ} \mathrm{C}$ for further use. 
The DNA samples were then processed for 16S rRNA sequencing by the Next Generation Sequencing Service of the Veterinary Diagnostic Lab (Kansas State University). The V3/V4 hypervariable region of the 16S rRNA gene was sequenced as specified by the manufacturer (Illumina, San Diego, CA, USA). Libraries were sequenced on an Illumina Miseq. Raw reads were trimmed for quality using FastQC v0.11.9 and analyzed using Mothur v1.44.1 [27-29]. The unique 16S reads, the output of Mothur (operational taxonomic units (OTUs)), were aligned to reference sequences from the SILVA rRNA database [30]. Near-identical sequences were merged using VSEARCH v2.15.1 [31].

\subsection{Fecal Microbiota Transplantation}

Fecal microbiota transplantation (FMT) was performed to determine the effect of the gut microbiota on the prevention of lung cancer in orthotopic LLC cell allografted mice. Four treatment regimens were performed: (1) PBS control, (2) 150-250 mg/kg of bEWE for 5 weeks prior to and 3 weeks after LLC cell injection, (3) FMT for 2 weeks prior to and 3 weeks after LLC cell injection, (4) 150-250 mg/ $\mathrm{kg}$ of bEWE treatment throughout the experiment along with antibiotics (ABX) for 2 weeks prior to and 3 weeks after LLC cell injection.

After 3 weeks of bEWE administration via drinking water, feces were collected under sterile conditions and stored at $-80{ }^{\circ} \mathrm{C}$ until further use for FMT. About 5-6 fecal pellets (about $100 \mathrm{mg}$ ) were thawed on ice and re-suspended in $1 \mathrm{~mL}$ Milli-Q water. The solution was vigorously mixed for $30 \mathrm{~s}$ by vortex and then centrifuged at $800 \times g$ for 3 min. About 500-600 $\mu \mathrm{L}$ of supernatant was collected and administered by oral gavage $(100 \mu \mathrm{L} /$ mouse/day) immediately after the collection [32]. Depletion of the gut microbiota was performed using a cocktail of ABX consisting of the following: ampicillin $(1 \mathrm{~g} / \mathrm{L})$ (USBiological Life Sciences, Salem, MA, USA), kanamycin (1 g/L) (Thermo Scientific, Waltham, MA, USA), metronidazole ( $1 \mathrm{~g} / \mathrm{L}$ ) (Cayman Chemical Company, Ann Arbor, MI, USA), and vancomycin (0.5 g/L) (Cayman Chemical Company, Ann Arbor, MI, USA) as previously described $[33,34]$. ABX treatment was conducted out via oral gavage (100 $\mu \mathrm{L} /$ mouse/day).

\subsection{Statistical Analysis}

The diversity of microbial populations within samples (alpha diversity) was assessed using the Chao1 estimator and observed indices. Comparison of microbial communities between samples (beta diversity) was evaluated using Principal coordinate analysis plots (PCoA), based on Bray-Curtis distances which demonstrated the dissimilarity of microbiota between samples and treatments. This was compared using the nonparametric analysis of similarities (ANOSIM) test. Microbiota statistical analysis was carried out using MicrobiomeAnalyst software [35].

All values are expressed as the mean \pm standard deviation of the mean. For all the experiments, statistical significance was assessed by an unpaired $t$-test and was conducted with multiple sample determinations. A $p$-value of $<0.05$ was considered statistically significant.

\section{Results}

3.1. Pretreatment with EWE and bEWE via Drinking Water Attenuated the Growth of Orthotopic Lung Carcinoma in Mice

The oral administration of water extracts from E. gracilis (EWE, bEWE, and PWE) via drinking water in mice was performed to evaluate their possible antitumor effects (Figure 1A). Body weights and water consumption were monitored every three days throughout the study period, and these were relatively consistent (Figures S1 and S2). The average body weights at the end of the study period were $20.6 \pm 0.6,20.6 \pm 0.7$, $20.6 \pm 0.6$, and $20.5 \pm 0.7 \mathrm{~g}$ with PBS, EWE, bEWE, and PWE treatment, respectively. Water consumption was $3.8 \pm 0.3,4.2 \pm 0.2,4.4 \pm 0.3,4 \pm 0.3 \mathrm{~mL} / \mathrm{mouse} /$ day with PBS, EWE, bEWE, and PWE treatment, respectively. As shown in Figure 1B, tumor growth was attenuated in the group pretreated with an average of $202 \pm 12 \mathrm{mg} / \mathrm{kg} /$ day EWE 
(tumor weight $=107.0 \pm 103.0 \mathrm{mg}, p>0.05$ ) as compared with the PBS control group (tumor weight $=203.2 \pm 91.6 \mathrm{mg}$ ). Treatment with an average of $213.1 \pm 13.3 \mathrm{mg} / \mathrm{kg} / \mathrm{day}$ bEWE further attenuated the growth of LLC tumors in the lungs of the mice (tumor weight $=19.9 \pm 31.3 \mathrm{mg}, p<0.05$ ). However, this attenuation was not observed in the group treated with an average of $19.7 \pm 2 \mathrm{mg} / \mathrm{kg} /$ day PWE (tumor weight $=152.8 \pm 199 \mathrm{mg}, p>0.05$ ). Although the dosage of the PWE is $1 / 10$ of those of EWE or bEWE, this is reflective of the yield of PWE from the same amount of Euglena gracilis. As shown in Figure 1C, the EWEand bEWE-treated mice developed fewer and smaller tumor nodules as compared to the PBS control group. Either EWE or bEWE treatment via drinking water did not alter mouse growth as measured by body weight (Figure S1). These results indicate that Euglena water extracts (EWE and bEWE) are effective in inhibiting the lung carcinoma growth in mice. A noteworthy finding in this study is that the bEWE is significantly more effective than the EWE in inhibiting lung tumor growth. This suggests that the heat treatment of EWE for $10 \mathrm{~min}$ in boiling water may be an important step in recovering the anticancer activity of Euglena gracilis.

\subsection{Euglena Water Extracts Alter the Gut Microbiota Composition}

Euglena water extracts (EWE and bEWE) reduce lung carcinoma growth in mice (Figure 1), which concurs with our recent study [19]. Since Euglena water extracts were administered daily via drinking water, there is a possibility of alteration of intestinal microbiota and/or their metabolisms. We, therefore, analyzed the gut microbiome using $16 S$ rRNA sequencing with the Illumina MiSeq platform in feces from the following groups: PBS (control), mice treated with EWE, and mice treated with bEWE, at three different time points (Day 0: before any treatment, Day 21: before LLC cell injection, Day 42: after LLC cell injection).

At a 97\% similarity level, we obtained 25620 OTUs and 11 phyla, 53 families, 104 genera after statistical analysis of $16 \mathrm{~S}$ rRNA sequences. Statistical analyses including alpha and beta diversity were quantified using observed index and Chao1 index and a principal coordinates analysis (PCoA), respectively. Chao1 estimates the total richness of the microbial community in a sample [36,37]. Our results showed no significant difference in alpha diversity between EWE- or bEWE-treated LLC tumor-bearing mice as compared to the PBS-treated group at both Day 21 and Day 42 (Figure 2A).

Beta diversity with principal coordinate analysis (PCOA) showed that bEWE- and EWE-treated mice had significantly different gut microbiome compositions (Figure 2B. ANOSIM, $\mathrm{R}=0.51, p<0.01$ ) as compared with PBS treated mice at Day 42 (ANOSIM, $\mathrm{R}=0.18, p<0.01$ ); however, some overlap between EWE and bEWE was observed, likely because both of these extracts were prepared from dried Euglena powder. ANOSIM based on Bray-Curtis distance indicated notable differences in microbial communities recovered from the fecal samples of LLC tumor-bearing mice treated with PBS, EWE, and bEWE.

\subsection{Euglena Water Extracts Caused Alteration in the Gut Microbial Communities}

A taxon-dependent analysis was conducted to explore the gut flora phylum and genus that potentially mediate or are closely associated with the attenuation of lung carcinoma growth upon Euglena water extracts administration. At the phylum level, the analysis showed the Bacteroidota and Firmicutes were the most abundant phyla identified. We observed that Bacteroidota abundance was significantly higher but Firmicutes abundance was significantly lower in the groups treated with Euglena extracts at Day 42 (after LLC cell inoculation) than in the PBS-treated group (Figure 3B,G). Furthermore, bEWE treatment at Day 42 was associated with a significantly higher abundance of Actinobacteriota and Proteobacteria when compared to PBS treatment (Figure 3A,H). We also observed a significantly higher abundance of Verrucomicrobiota, but a significantly lower abundance of Deferribacterota, in groups treated with Euglena water extracts at Day 42, when compared to the PBS treated group (Figure 3I,E). Currently, Akkermansia muciniphila is the only identified representative species of the phylum Verrucomicrobiota [38], and Akkermansia 
abundance was also significantly higher with EWE or bEWE treatment (Figure 4A). Further, significantly higher abundance of Muribaculum (phylum Bacteroidota) and significant decrease of Mucispirillum (phylum Deferribacterota) was observed at Day 42 with EWE or bEWE treatment, rather than with PBS treatment (Figure 4B,C).
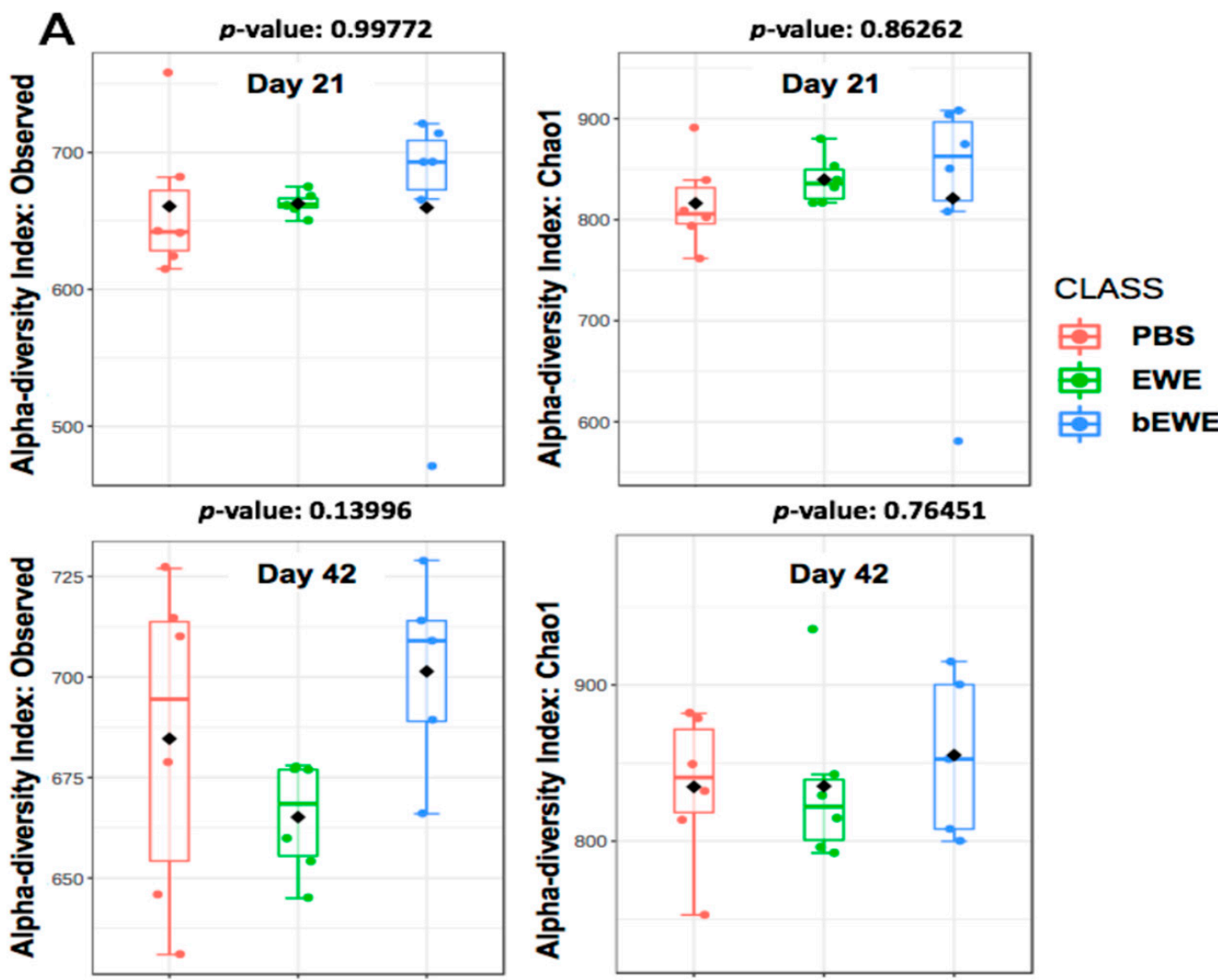

B

Day 0

R:0.17942; p-value $<0.008$

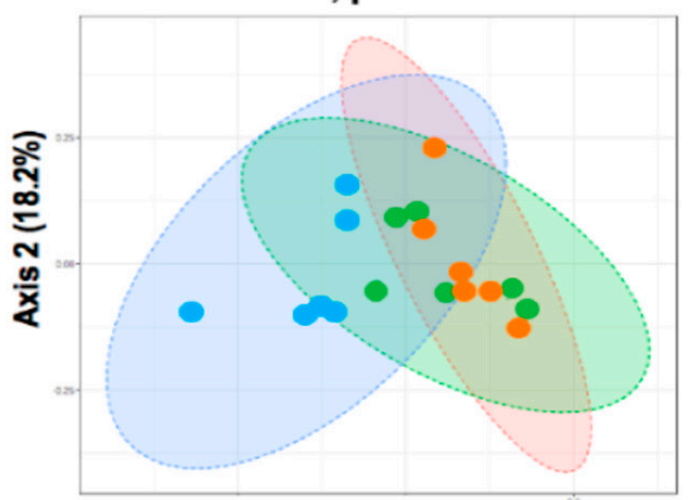

Axis $1(18.4 \%)$

Day 42

R:0.51042; p-value $<0.002$

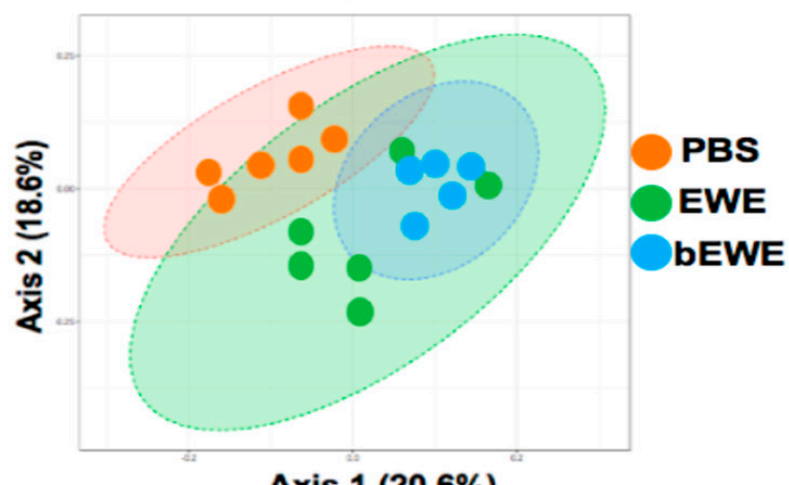

Axis 1 (20.6\%)

Figure 2. Alpha and beta diversity of gut microbiota in Euglena water extracts and PBS treated mouse feces before (Day 21) and after LLC cell inoculation (Day 42). (A) The difference in alpha diversity; measured as observed index and Chao1 index for Day 21 (upper panels in panel A) and Day 42 (lower panels in panel A). Box-and-whisker plots show high, low, and median values, with lower and upper edges of each box denoting first and third quartiles, respectively. (B) Principal coordinates analysis (PCoA) based on Bray-Curtis dissimilarity representing the difference in gut microbial communities. Each PCoA plot is accompanied by an analysis of similarity (ANOSIM) using an appropriate distance matrix. The R-values closer to zero indicates no difference between groups. 

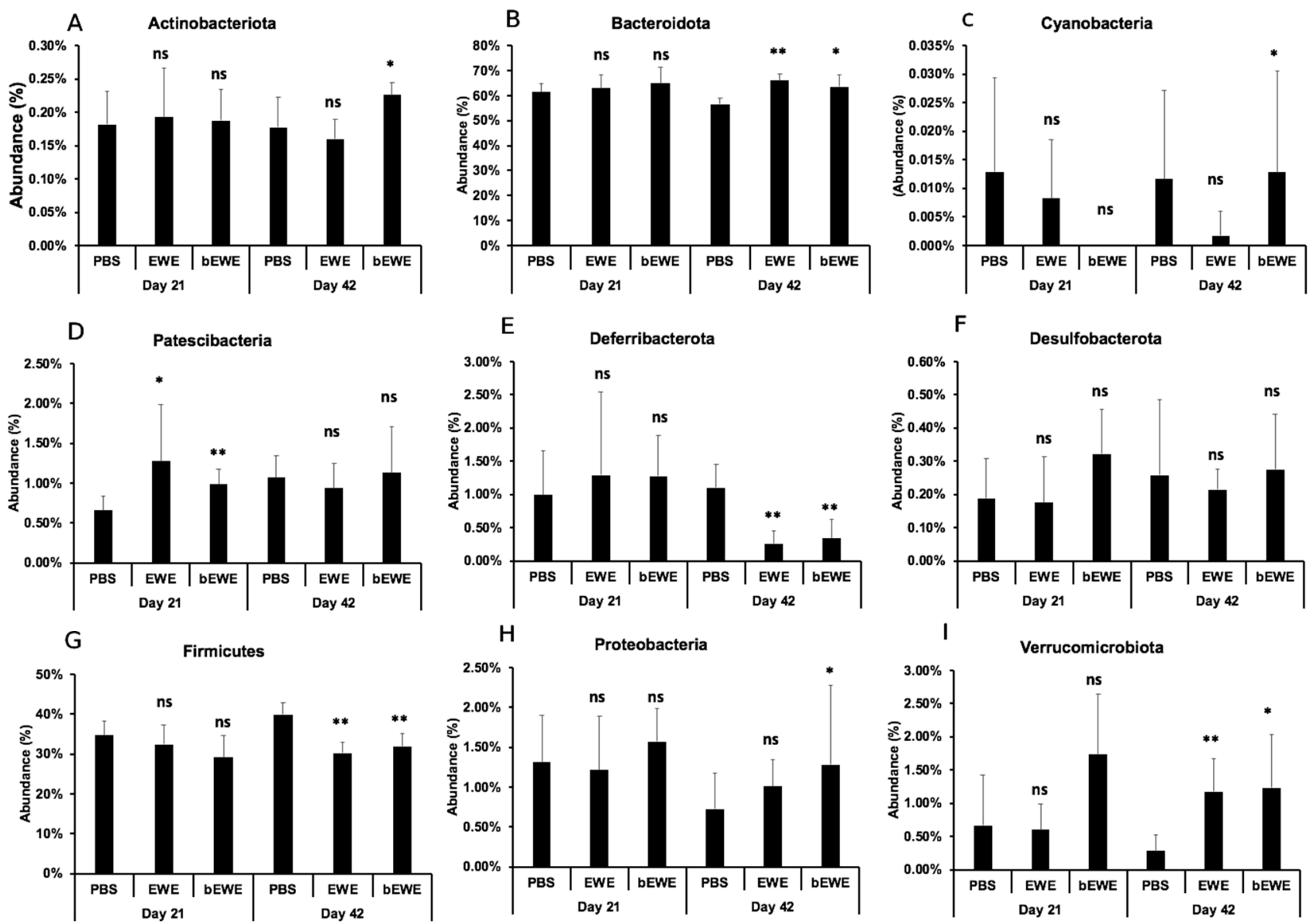

Figure 3. Relative abundance of bacterial taxa at the phylum level in mice treated with Euglena water extracts at Day 21 (before LLC cell inoculation) and Day 42 (after LLC cell inoculation). Percentage of taxa abundance of (A) Actinobacteriota, (B) Bacteroidota, (C) Cyanobacteria, (D) Patescibacteria, (E) Deferribacterota, (F) Desulfobacterota, (G) Firmicutes, (H) Proteobacteria, and (I) Verrucomicrobiota is presented. The difference in taxa abundance for each phylum between PBS and EWE or bEWE was compared at Day 21 and Day 42 using a $t$-test. Results are presented as mean \pm SD $(n=5-6) .{ }^{*}: p<0.05 ; * *: p<0.01$; ns: $p>0.05$.
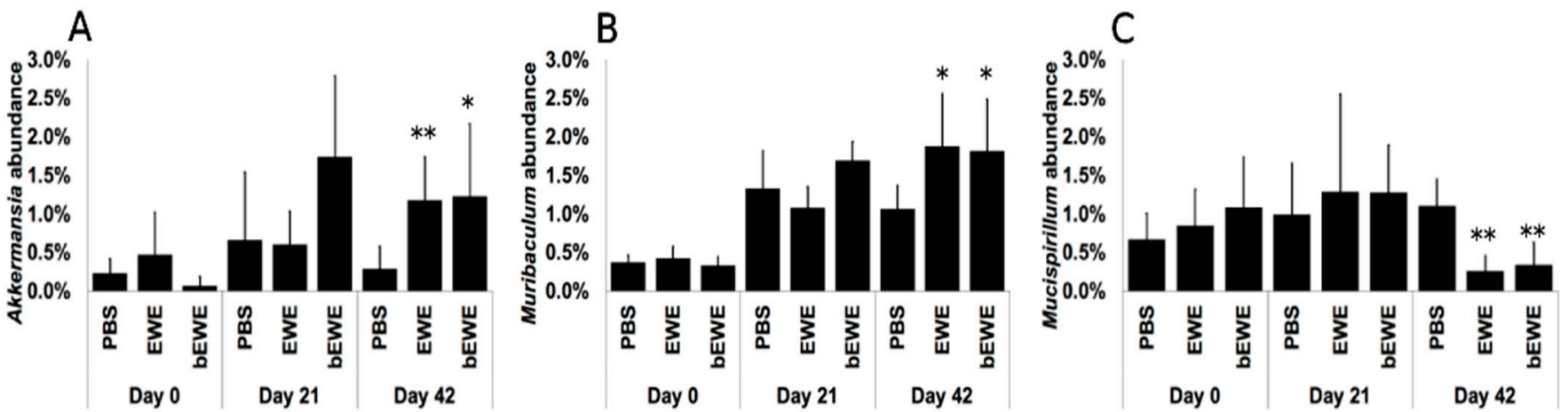

Figure 4. Relative abundance of bacterial taxa at the genus level in mice treated with Euglena water extracts at Day 21 (before LLC cell inoculation) and Day 42 (after LLC cell inoculation). Percentage of taxa abundance of (A) Akkermansia, (B) Muribaculum, and (C) Mucispirillum between PBS and EWE or bEWE were compared at Day 21 and Day 42 using $t$-test. Results are presented as mean \pm SD $(n=5-6) .{ }^{*}: p<0.05 ;{ }^{* *}: p<0.01$. 
3.4. Transplantation of Fecal Microbiota from EWE-Treated Mice Resulted in Attenuation of Lung Cancer Growth

To further understand the role of microbiota in the prevention of lung carcinoma growth in mice by daily oral treatment with Euglena extract treated by boiling (bEWE) (Group \#1 in Figure 5A), experiments with fecal microbiota transplantation (FMT) (Group $\# 2$ in Figure 5A) and antibiotics treatment (ABX) (Group \#3 in Figure 5A) were performed as shown in Figure 5A. After 3 weeks of bEWE administration via drinking water, feces were collected and used for FMT for 5 weeks (Group \#2 in Figure 5A). From this experiment, we observed that FMT-treatment significantly attenuated the growth of tumors in the mouse lung $(349.3 \pm 232.0 \mathrm{mg}, p<0.05)$ as compared with the PBS control group $(591.2 \pm 194.1 \mathrm{mg})$. The FMT-induced reduction of the lung tumor growth was similar to that of bEWE treated mice, which also experienced a significant decrease in the tumor burden $(289.9 \pm 98.1 \mathrm{mg}, p<0.05)$ in comparison to the burden of the PBS groups. On the contrary, the ABX treatment reversed the bEWE treatment-induced tumor growth attenuation and the tumor burden $(452.7 \pm 302.9 \mathrm{mg})$ was similar to that of the control group. Although a little variation in body weight was observed between FMT and ABX groups (administered through oral gavage) and PBS and bEWE groups (administered via drinking water, Figure S3), these variations were not statistically significant. These results strongly suggest that the intestinal microbiota plays a role in the attenuation of lung carcinoma growth in mice by oral administration of bEWE.

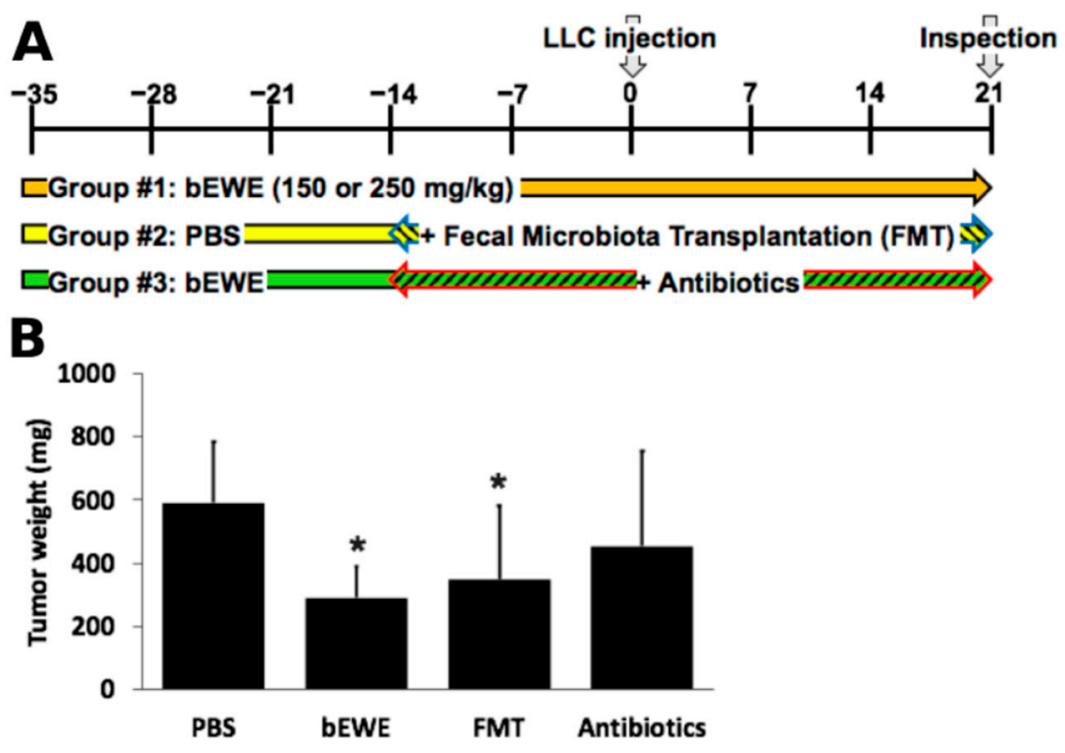

Figure 5. Fecal microbiota transplantation (FMT) from bEWE treated mice significantly attenuated the growth of tumors in the murine lung. (A) Schematic illustration of the study design. (B) Average tumor weight in each treatment group. PBS served as control. Results are presented as mean \pm SD $(n=5){ }^{*}: p<0.05$ as compared to PBS-treated group.

\section{Discussion}

Compounds present in organisms of the genus Euglena have considerable potential as pharmaceuticals and nutraceuticals. Since Euglena gracilis is easy to cultivate and has a faster growth rate than other Euglenophyceae species, it is widely used in basic research as a model organism [9]. Although several studies have been conducted to determine the therapeutic properties of substances obtained from Euglena against various diseases, including cancers $[14,15,39,40]$, their effect on the gut microbiota is still not well understood. In the present study, we investigated the effect of oral daily administration of E. gracilis water extracts devoid of paramylons on the gut microbiota of mice and its association with the attenuation of lung cancer growth. Pretreatment with two types of Euglena water extracts (EWE and bEWE) via drinking water attenuated the growth of orthotopic lung 
carcinoma in syngeneic mice (Figure 1B). Among two types of extract, bEWE (EWE with boiling)-induced tumor growth attenuation was much stronger than that by EWE. This result indicates that heat treatment and boiling for $10 \mathrm{~min}$ altered either the quality or quantity of antitumor substances in the EWE. Although it is difficult to pinpoint the specific mechanism by which the heat treatment increased the antitumor effect of EWE, due to the unknown chemical nature of this antitumor substance it is conceivable that boiling may have resulted in increasing the solubility and diffusion rate of the bioactive molecules, which could lead to their increased absorption and efficiency. Alternatively, boiling and the subsequent centrifugation may have removed a potential inhibitor against antitumor substances in the EWE. Indeed, large denatured molecules have been removed by this process (data not shown). Nonetheless, this experiment warrants a follow-up study to identify the antitumor substance(s) present in Euglena extracts.

The mouse study revealed that FMT using stools from bEWE-fed mice attenuated lung carcinoma growth at the same level as in mice who were orally administered bEWE (Figure 5B). We observed that the administration of Euglena water extracts (bEWE and EWE) increased the ratio of Bacteroidetes to Firmicutes along with a significant increase in the genera Akkermansia and Muribaculum in lung tumor-bearing mice (Figures 3B,G and 4). These results suggest that oral administration of partially purified water extracts from $E$. gracilis alters the intestinal microbiota and induces a significant suppression of lung carcinoma growth, presumably due to changes in the bacteria itself and/or their metabolites.

The association between gut microbiota and lung cancer has received increased appreciation after recent studies indicated the involvement of the gut-lung axis in the development of cancers by altering the immune system [41]. In the present study, we found no significant difference in the alpha diversity of gut microbiota in mice treated with Euglena water extracts in comparison to the PBS-treated mice. However, the beta diversity analysis showed a significant difference in the microbial composition (Figure 2). It has been reported that the gut microbial composition in healthy humans is significantly different from that in patients with cancers, including lung cancer [25,41,42]. Therefore, an alteration of the gut microbiota composition appears to be significantly influential on carcinogenesis in the lung.

In the current study, we observed significant alterations in the gut microbiota at Day 42 (end of the study period) after LLC cell injection in mice treated with Euglena water extracts (Figure 3). The alterations were characterized as a significant increase in the relative abundance of microbes of the phyla Bacteroidota, Verrucomicrobiota, Actinobacteriota, and Proteobacteria, while there was a significant reduction for the phyla Firmicutes and Deferribacterota (Figure 3). Since bacteria of the phyla Bacteroidota and Firmicutes are most abundant in the gut of humans and mice, any alteration in these bacteria potentially disrupts immunological homeostasis, thereby leading to various disease conditions. In support of this notion, an increase has been reported in the ratio of Firmicutes to Bacteroidota in patients with irritable bowel syndrome (IBS), autism, hypertension, chronic fatigue syndrome, and cancer patients, in comparison to healthy humans [43-46]. Similarly, another study found a higher ratio of Firmicutes to Bacteroidota in lung cancer patients than in healthy controls [47]. The current study found that administration of Euglena water extracts significantly decreased lung tumor growth and the ratio of Firmicutes to Bacteroidota. Therefore, it is suggested that daily oral administration of Euglena water extracts induces an attenuation of lung carcinoma growth, at least in part by the alteration of the gut microbial ratios of Firmicutes and Bacteroidota.

The current study also found that the abundance of organisms of phylum Deferribacteres and genus Mucispirillum was significantly lower in the LLC tumor-bearing mice treated with the Euglena water extracts at Day 42 (Figures 3E and 4C). Deferribacteres are intestinal flora that play a role in iron metabolism and are associated with bowel iron balance [48]. Increased iron metabolism is associated with malignant transformation and cancer progression, as cancer cells are highly dependent on iron compared to normal cells $[49,50]$. Therefore, higher iron metabolism increases the risk of cancers and promotes tumor growth [48]. Although the underlying mechanisms are still not clear, imbalances in 
pulmonary iron levels are associated with the development of lung cancers [51]. Results in the current study suggest that Euglena water extract-induced growth attenuation of lung cancer is possibly associated also with the disruption of iron metabolism.

In our study, the abundance of genera Akkermansia and Muribaculum was found to be significantly higher in mice treated with Euglena water extracts than those treated with PBS at Day 42 (Figure 4). Although the study did not include the species-level identification within Akkermansia and Muribaculum, the significant increase in abundance for these genera maybe linked to the attenuation of lung carcinoma growth. Currently, Akkermansia muciniphila is the only representative species of the phylum Verrucomicrobiota, which typically reside in the large intestine of humans and other animals [38]. A. muciniphila has been reported as a beneficial microorganism due to its displayed role in increasing anti-inflammatory functions, improving host metabolism, and enhancing cancer treatment $[52,53]$. A human study revealed that $A$. muciniphila is less abundant in patients with lung cancer and supplementation of $A$. muciniphila improves the efficacy of immune checkpoint inhibitor (PD-1 blockade) therapy in lung, skin, and renal cancer treatments [54]. In addition, the present study also found that the abundance of Muribaculum, a genus belonging to the phylum Bacteroidota, was increased in the LLC tumor-bearing mice treated with Euglena water extracts (Figure 4B). Because an increase in Muribaculum is associated with better therapeutic efficacy of natural compounds in LLC tumor-bearing mice [55], it is suggested that Akkermansia and Muribaculum are the gut bacteria that could play a significant role in the Euglena water extracts-induced attenuation of lung carcinoma growth in mice.

\section{Conclusions}

In summary, the present study revealed that oral administration of Euglena water extracts (EWE and bEWE) prevents lung cancer growth in an orthotopic syngeneic mouse model. Daily oral administration of Euglena water extracts caused significant alteration in the gut microbiota, including an increase in the ratio of Bacteroidota to Firmicutes, an increase in Verrucomicrobiota, a decrease of Deferribacteres, and an increase of the genera Akkermansia and Muribaculum. The FMT and ABX studies confirmed that the attenuation of lung carcinoma growth in mice by Euglena water extracts is associated with the alteration of the gut microbiota. The alteration of the gut microbiome may induce microbial metabolite-dependent suppression in cancer growth inhibition. Future studies are needed to identify the bacterial metabolites involved in lung cancer growth attenuation and the mechanisms by which they attenuate the growth of lung cancer. The current study strongly suggests that oral daily administration of Euglena water extracts alters the gut microbiota, thereby attenuating lung carcinoma growth. This study indicates that the water extract from E. gracilis is a promising prebiotic for the prevention of lung cancer. This study also provides insights for developing a new rationale for lung cancer prevention strategy which would be of great value for human health.

Supplementary Materials: The following are available online at https: / www.mdpi.com/article / $10.3390 /$ nu14030678/s1, Figure S1. Treatment of mice with water extracts from E. gracilis didn't alter the body weight. Body weight was $20.6 \pm 0.6,20.6 \pm 0.7,20.6 \pm 0.6$, and $20.5 \pm 0.7 \mathrm{~g}$ in average with PBS, EWE, bEWE, and PWE treatments, respectively; Figure S2. Consumption of water with different treatments was relatively consistent throughout the study. Water consumption were $3.8 \pm 0.3,4.2 \pm 0.2,4.4 \pm 0.3,4 \pm 0.3 \mathrm{~mL} /$ mouse/day with PBS, EWE, bEWE, and PWE treatments, respectively; Figure S3. The body weight of mice treated with PBS, bEWE, FMT, or antibiotics was comparable. The average body weights at the end of the study period were 19.9 $\pm 1.0,19.8 \pm 1.0$, $18.9 \pm 0.6$, and $19.4 \pm 0.6 \mathrm{~g}$ with PBS, bEWE, FMT, and bEWE + Antibiotics treatments, respectively.

Author Contributions: Conceptualization: M.T. Resources: A.N., K.S. Methodology: D.U., S.I., N.R., M.T. Investigation: D.U., S.I., N.R., M.T. Validation and Formal Analysis: D.U., S.I. Data Curation: D.U., S.I., M.T. Writing-Original Draft Preparation, Writing-Review \& Editing and Visualization: D.U., S.I., J.C., M.T. Supervision, Project Administration and Funding Acquisition: J.C., M.T. All authors have read and agreed to the published version of the manuscript. 
Funding: This work was supported in part by Kansas State University (KSU) College of Veterinary Medicine Dean's funds [2018 CVM-SMILE] (MT), Euglena Research Fund (JC, MT), KSU Johnson Cancer Research Center 2020 JCRC-CRA (MT), K-INBRE Bridging Award [P20 GM103418] (MT), and NSF grant DMR-1945589 (JC).

Institutional Review Board Statement: All experiments were carried out under approvals by the Institutional Animal Care and Use Committee (Protocol \# 4346) and Institutional Biosafety Committee (Registration \# 1317) of the Kansas State University.

Informed Consent Statement: Not applicable.

Data Availability Statement: All data is available upon request to the corresponding author.

Acknowledgments: We thank Rachel Palinski (Next-generation sequencing lab, Kansas State University) for her technical support on the $16 \mathrm{~S}$ rRNA gene sequencing analysis. We also thank Morgan Phillips (Kansas State University) for proofreading the manuscript.

Conflicts of Interest: No potential conflict of interest was disclosed by all authors.

\section{References}

1. Bray, F.; Ferlay, J.; Soerjomataram, I.; Siegel, R.L.; Torre, L.A.; Jemal, A. Global cancer statistics 2018: GLOBOCAN estimates of incidence and mortality worldwide for 36 cancers in 185 countries. CA A Cancer J. Clin. 2018, 68, 394-424. [CrossRef]

2. Samet, J.M.; Avila-Tang, E.; Boffetta, P.; Hannan, L.M.; Olivo-Marston, S.; Thun, M.J.; Rudin, C.M. Lung Cancer In Never Smokers: Clinical Epidemiology And Environmental Risk Factors. Clin. Cancer Res. Off. J. Am. Assoc. Cancer Res. 2009, 15, 5626-5645. [CrossRef] [PubMed]

3. Torok, S.; Hegedus, B.; Laszlo, V.; Hoda, M.A.; Ghanim, B.; Berger, W.; Klepetko, W.; Dome, B.; Ostoros, G. Lung cancer in never smokers. Future Oncol. 2011, 7, 1195-1211. [CrossRef]

4. $\quad$ Siegel, R.L.; Miller, K.D.; Fuchs, H.E.; Jemal, A. Cancer Statistics. CA A Cancer J. Clin. 2021, 71, 7-33. [CrossRef]

5. Stella, G.M.; Kolling, S.; Benvenuti, S.; Bortolotto, C. Lung-Seeking Metastases. Cancers 2019, 11, 1010. [CrossRef]

6. Suzuki, K. Large-Scale Cultivation of euglena. In Euglena: Biochemistry, Cell and Molecular Biology; Springer: Berlin/Heidelberg, Germany, 2017; pp. 285-293. [CrossRef]

7. Zakryś, B.; Milanowski, R.; Karnkowska, A. Evolutionary origin of euglena. In Euglena: Biochemistry, Cell and Molecular Biology; Springer: Berlin/Heidelberg, Germany,2017; pp. 3-17. [CrossRef]

8. Gissibl, A.; Sun, A.; Care, A.; Nevalainen, H.; Sunna, A. Bioproducts From Euglena gracilis: Synthesis and Applications. Front. Bioeng. Biotechnol. 2019, 7, 108. [CrossRef]

9. Kottuparambil, S.; Thankamony, R.L.; Agusti, S. Euglena as a potential natural source of value-added metabolites. A review. Algal Res. 2019, 37, 154-159. [CrossRef]

10. Kondo, Y.; Kato, A.; Hojo, H.; Nozoe, S.; Takeuchi, M.; Ochi, K. Cytokine-Related Immunopotentiating Activities of Paramylon, a $\beta-(1 \rightarrow 3)-D-G l u c a n$ from Euglena gracilis. J. Pharm. Dyn. 1992, 15, 617-621. [CrossRef]

11. Russo, R.; Barsanti, L.; Evangelista, V.; Frassanito, A.M.; Longo, V.; Pucci, L.; Penno, G.; Gualtieri, P. Euglena gracilis paramylon activates human lymphocytes by upregulating pro-inflammatory factors. Food Sci. Nutr. 2017, 5, 205-214. [CrossRef] [PubMed]

12. Sakagami, H.; Kikuchi, K.; Takeda, M.; Sato, T.; Ichikawa, S.; Fujimaki, M.; Wada, C.; Komatsu, N. Macrophage stimulation activity of antimicrobial N,N-dimethylaminoethyl paramylon. In Vivo 1991, 5, 101-105. [PubMed]

13. Sugiyama, A.; Suzuki, K.; Mitra, S.; Arashida, R.; Yoshida, E.; Nakano, R.; Yabuta, Y.; Takeuchi, T. Hepatoprotective Effects of Paramylon, a $\beta-1,3-D-G l u c a n$ Isolated from Euglena gracilis Z, on Acute Liver Injury Induced by Carbon Tetrachloride in Rats. J. Vet. Med. Sci. 2009, 71, 885-890. [CrossRef]

14. Watanabe, T.; Shimada, R.; Matsuyama, A.; Yuasa, M.; Sawamura, H.; Yoshida, E.; Suzuki, K. Antitumor activity of the $\beta$-glucan paramylon from Euglena against preneoplastic colonic aberrant crypt foci in mice. Food Funct. 2013, 4, 1685. [CrossRef]

15. Nakashima, A.; Suzuki, K.; Asayama, Y.; Konno, M.; Saito, K.; Yamazaki, N.; Takimoto, H. Oral administration of Euglena gracilis $\mathrm{Z}$ and its carbohydrate storage substance provides survival protection against influenza virus infection in mice. Biochem. Biophys. Res. Commun. 2017, 494, 379-383. [CrossRef] [PubMed]

16. Nakashima, H.; Ohshiro, Y.; Miyano, N.; Yamamoto, N.; Ichikawa, S.; Kondo, H.; Takeda, M.; Sakagami, H. Synergistic inhibition of human immunodeficiency virus type 1 (HIV-1) replication in vitro by sulphated paramylon and $3^{\prime}$-azido-2' $3^{\prime}$-dideoxythymidine (AZT). Lett. Appl. Microbiol. 1994, 18, 24-26. [CrossRef]

17. Nakashima, A.; Horio, Y.; Suzuki, K.; Isegawa, Y. Antiviral Activity and Underlying Action Mechanism of Euglena Extract against Influenza Virus. Nutrients 2021, 13, 3911. [CrossRef]

18. Shimada, R.; Fujita, M.; Yuasa, M.; Sawamura, H.; Watanabe, T.; Nakashima, A.; Suzuki, K. Oral administration of green algae, Euglena gracilis, inhibits hyperglycemia in OLETF rats, a model of spontaneous type 2 diabetes. Food Funct. 2016, 7, 4655-4659. [CrossRef] 
19. Ishiguro, S.; Upreti, D.; Robben, N.; Burghart, R.; Loyd, M.; Ogun, D.; Le, T.; Delzeit, J.; Nakashima, A.; Thakkar, R.; et al. Water extract from Euglena gracilis prevents lung carcinoma growth in mice by attenuation of the myeloid-derived cell population. Biomed. Pharmacother. 2020, 127, 110166. [CrossRef]

20. Hillman, E.T.; Lu, H.; Yao, T.; Nakatsu, C.H. Microbial Ecology along the Gastrointestinal Tract. Microbes Environ. 2017, 32, 300-313. [CrossRef] [PubMed]

21. Budden, K.F.; Gellatly, S.L.; Wood, D.L.A.; Cooper, M.A.; Morrison, M.; Hugenholtz, P.; Hansbro, P.M. Emerging pathogenic links between microbiota and the gut-lung axis. Nat. Rev. Microbiol. 2017, 15, 55-63. [CrossRef] [PubMed]

22. Dang, A.T.; Marsland, B.J. Microbes, metabolites, and the gut-lung axis. Mucosal Immunol. 2019, 12, 843-850. [CrossRef] [PubMed]

23. den Besten, G.; van Eunen, K.; Groen, A.K.; Venema, K.; Reijngoud, D.-J.; Bakker, B.M. The role of short-chain fatty acids in the interplay between diet, gut microbiota, and host energy metabolism. J. Lipid Res. 2013, 54, 2325-2340. [CrossRef]

24. Zhang, D.; Li, S.; Wang, N.; Tan, H.-Y.; Zhang, Z.; Feng, Y. The Cross-Talk Between Gut Microbiota and Lungs in Common Lung Diseases. Front. Microbiol. 2020, 11, 301. [CrossRef] [PubMed]

25. Zhuang, H.; Cheng, L.; Wang, Y.; Zhang, Y.-K.; Zhao, M.-F.; Liang, G.-D.; Zhang, M.-C.; Li, Y.-G.; Zhao, J.-B.; Gao, Y.-N.; et al. Dysbiosis of the Gut Microbiome in Lung Cancer. Front. Cell. Infect. Microbiol. 2019, 9, 112. [CrossRef]

26. Nakashima, A.; Sasaki, K.; Sasaki, D.; Yasuda, K.; Suzuki, K.; Kondo, A. The alga Euglena gracilis stimulates Faecalibacterium in the gut and contributes to increased defecation. Sci. Rep. 2021, 11, 1-8. [CrossRef] [PubMed]

27. Schloss, P.D.; Westcott, S.L.; Ryabin, T.; Hall, J.R.; Hartmann, M.; Hollister, E.B.; Lesniewski, R.A.; Oakley, B.B.; Parks, D.H.; Robinson, C.J.; et al. Introducing mothur: Open-Source, Platform-Independent, Community-Supported Software for Describing and Comparing Microbial Communities. Appl. Environ. Microbiol. 2009, 75, 7537-7541. [CrossRef] [PubMed]

28. Andrews, S. FastQC: A Quality Control Tool for High Throughput Sequence Data. 2010. Available online: http://www. bioinformatics.babraham.ac.uk/projects/fastqc/. (accessed on 22 December 2021).

29. Schloss, P.D. Reintroducing mothur: 10 Years Later. Appl. Environ. Microbiol. 2020, 86, e2343-19. [CrossRef]

30. Quast, C.; Pruesse, E.; Yilmaz, P.; Gerken, J.; Schweer, T.; Yarza, P.; Peplies, J.; Glöckner, F.O. The SILVA ribosomal RNA gene database project: Improved data processing and web-based tools. Nucleic Acids Res. 2013, 41, D590-D596. [CrossRef] [PubMed]

31. Rognes, T.; Flouri, T.; Nichols, B.; Quince, C.; Mahé, F. VSEARCH: A versatile open source tool for metagenomics. PeerJ 2016, 4, e2584. [CrossRef]

32. Lai, Z.-L.; Tseng, C.-H.; Ho, H.J.; Cheung, C.K.Y.; Lin, J.-Y.; Chen, Y.-J.; Cheng, F.-C.; Hsu, Y.-C.; Lin, J.-T.; El-Omar, E.M.; et al Fecal microbiota transplantation confers beneficial metabolic effects of diet and exercise on diet-induced obese mice. Sci. Rep. 2018, 8, 15625. [CrossRef] [PubMed]

33. Martínez-López, M.; Iborra, S.; Conde-Garrosa, R.; Mastrangelo, A.; Danne, C.; Mann, E.R.; Reid, D.M.; Gaboriau-Routhiau, V.; Chaparro, M.; Lorenzo, M.P.; et al. Microbiota Sensing by Mincle-Syk Axis in Dendritic Cells Regulates Interleukin-17 and -22 Production and Promotes Intestinal Barrier Integrity. Immunity 2019, 50, 446-461.e9. [CrossRef]

34. Guo, W.; Zhou, X.; Li, X.; Zhu, Q.; Peng, J.; Zhu, B.; Zheng, X.; Lu, Y.; Yang, D.; Wang, B.; et al. Depletion of Gut Microbiota Impairs Gut Barrier Function and Antiviral Immune Defense in the Liver. Front. Immunol. 2021, 12, 897. [CrossRef]

35. Dhariwal, A.; Chong, J.; Habib, S.; King, I.L.; Agellon, L.B.; Xia, J. MicrobiomeAnalyst: A web-based tool for comprehensive statistical, visual and meta-analysis of microbiome data. Nucleic Acids Res. 2017, 45, W180-W188. [CrossRef]

36. Chao, A. Estimating the population size for capture-recapture data with unequal catchability. Biometrics 1987, 43, 783-791. [CrossRef] [PubMed]

37. Wang, M.; Ahrnã $@$, S.; Jeppsson, B.; Molin, G. Comparison of bacterial diversity along the human intestinal tract by direct cloning and sequencing of 16S rRNA genes. FEMS Microbiol. Ecol. 2005, 54, 219-231. [CrossRef]

38. Naito, Y.; Uchiyama, K.; Takagi, T. A next-generation beneficial microbe: Akkermansia Muciniphila. J. Clin. Biochem. Nutr. 2018, 63, 33-35. [CrossRef] [PubMed]

39. Das, B.K.; Pradhan, J.; Pattnaik, P.; Samantaray, B.R.; Samal, S.K. Production of antibacterials from the freshwater alga Euglena viridis (Ehren). World J. Microbiol. Biotechnol. 2005, 21, 45-50. [CrossRef]

40. Quesada, L.A.; Lustig, E.S.; Marechal, L.R.; Belocopitow, E. Antitumor Activity of Paramylon on Sarcoma-180 in Mice. GANN Jpn. J. Cancer Res. 1976, 67, 455-459. [CrossRef]

41. Gui, Q.; Li, H.; Wang, A.; Zhao, X.; Tan, Z.; Chen, L.; Xu, K.; Xiao, C. The association between gut butyrate-producing bacteria and non-small-cell lung cancer. J. Clin. Lab. Anal. 2020, 34, e23318. [CrossRef]

42. Zhou, A.; Lei, Y.; Tang, L.; Hu, S.; Yang, M.; Wu, L.; Yang, S.; Tang, B. Gut microbiota: The emerging link to lung homeostasis and disease. J. Bacteriol. 2020, 203, e00454. [CrossRef]

43. Heshiki, Y.; Vazquez-Uribe, R.; Li, J.; Ni, Y.; Quainoo, S.; Imamovic, L.; Li, J.; Sørensen, M.; Chow, B.K.C.; Weiss, G.J.; et al Predictable modulation of cancer treatment outcomes by the gut microbiota. Microbiome 2020, 8, 1-14. [CrossRef]

44. Nagel, R.; Traub, R.J.; Allcock, R.J.N.; Kwan, M.M.S.; Bielefeldt-Ohmann, H. Comparison of faecal microbiota in Blastocystispositive and Blastocystis-negative irritable bowel syndrome patients. Microbiome 2016, 4, 47. [CrossRef]

45. Strati, F.; Cavalieri, D.; Albanese, D.; De Felice, C.; Donati, C.; Hayek, J.; Jousson, O.; Leoncini, S.; Renzi, D.; Calabrò, A.; et al. New evidences on the altered gut microbiota in autism spectrum disorders. Microbiome 2017, 5, 24. [CrossRef]

46. Yang, T.; Santisteban, M.M.; Rodriguez, V.; Li, E.; Ahmari, N.; Carvajal, J.M.; Zadeh, M.; Gong, M.; Qi, Y.; Zubcevic, J.; et al. Gut Dysbiosis Is Linked to Hypertension. Hypertension 2015, 65, 1331-1340. [CrossRef] [PubMed] 
47. Chau, J.; Yadav, M.; Furqan, M.; Cady, N.; Mercer, K.; Eastman, E.; Abu-Hejleh, T.; Clamon, G.; Shahi, S.; Dai, Q.; et al. P2.04-18 Analysis of Patient Microbiome and Its Correlation to Immunotherapy Response and Toxicity in Lung Cancer. J. Thorac. Oncol. 2019, 14, S715. [CrossRef]

48. Li, Y.; Luan, Y.; Yue, X.; Xiang, F.; Mao, D.; Cao, Y.; Xiong, Z. Effects of Codonopis bulleynana forest ex diels on Deferribacteres in constipation predominant intestine tumor: Differential analysis. Saudi J. Biol. Sci. 2018, 26, 395-401. [CrossRef] [PubMed]

49. Brown, R.A.M.; Richardson, K.L.; Kabir, T.D.; Trinder, D.; Ganss, R.; Leedman, P.J. Altered Iron Metabolism and Impact in Cancer Biology, Metastasis, and Immunology. Front. Oncol. 2020, 10, 476. [CrossRef]

50. Chen, Y.; Fan, Z.; Yang, Y.; Gu, C. Iron metabolism and its contribution to cancer (Review). Int. J. Oncol. 2019, 54, 1143-1154. [CrossRef]

51. Neves, J.; Haider, T.; Gassmann, M.; Muckenthaler, M.U. Iron Homeostasis in the Lungs-A Balance between Health and Disease. Pharmaceuticals 2019, 12, 5. [CrossRef]

52. Hu, X.; Zhao, Y.; Yang, Y.; Gong, W.; Sun, X.; Yang, L.; Zhang, Q.; Jin, M. Akkermansia muciniphila Improves Host Defense Against Influenza Virus Infection. Front. Microbiol. 2021, 11, 586476. [CrossRef] [PubMed]

53. Zhang, T.; Li, Q.; Cheng, L.; Buch, H.; Zhang, F. Akkermansia muciniphila is a promising probiotic. Microb. Biotechnol. 2019, 12, 1109-1125. [CrossRef] [PubMed]

54. Routy, B.; le Chatelier, E.; DeRosa, L.; Duong, C.P.M.; Alou, M.T.; Daillère, R.; Fluckiger, A.; Messaoudene, M.; Rauber, C.; Roberti, M.P.; et al. Gut microbiome influences efficacy of PD-1-based immunotherapy against epithelial tumors. Science 2018 359, 91-97. [CrossRef] [PubMed]

55. Huang, J.; Liu, D.; Wang, Y.; Liu, L.; Li, J.; Yuan, J.; Jiang, Z.; Jiang, Z.; Hsiao, W.W.; Liu, H.; et al. Ginseng polysaccharides alter the gut microbiota and kynurenine/tryptophan ratio, potentiating the antitumour effect of antiprogrammed cell death 1/programmed cell death ligand 1 (anti-PD-1/PD-L1) immunotherapy. Gut 2021, gutjnl-2020-321031. [CrossRef] [PubMed] 